\title{
Adipose-Tissue Pyruvate Kinase
}

\section{PROPERTIES AND INTERCONVERSION OF TWO ACTIVE FORMS}

\author{
By C. I. POGSON \\ Molecular Enzymology Laboratory, Department of Biochemistry, University of Bristol
}

\author{
(Received 20 May 1968)
}

\begin{abstract}
1. Extraction of rat epididymal adipose tissue with buffer containing EDTA yields a pyruvate kinase, provisionally called PyK-A, the properties of which resemble in several respects those of the allosteric pyruvate kinase of liver. These properties include co-operative interactions with phosphoenolpyruvate, $\mathrm{Mg}^{2+}, \mathrm{K}^{+}, \mathrm{NH}_{4}{ }^{+}$ and ATP, and sensitivity to activation by fructose 1,6-diphosphate. 2. Extraction in the absence of EDTA yields predominantly a form, PyK-B, that shows both normal Michaelis-Menten kinetics with phosphoenolpyruvate, $\mathrm{Mg}^{2+}$ and $\mathrm{ATP}$. and co-operative interactions with $\mathrm{K}^{+}$and $\mathrm{NH}_{4}{ }^{+}$; this form is insensitive towards fructose 1,6-diphosphate. 3. Both forms yield simple kinetics with ADP, though $K_{m}$ values differ in the two systems. In all cases where co-operativity has been demonstrated, Hill-plot $n$ values are between $1 \cdot 4$ and 2.0. 4. The conversion of PyK-A into PyK-B is mediated specifically by fructose 1,6-diphosphate; the reverse reaction is occasioned by EDTA, ATP or citrate. It is thought that a bivalent cation may be involved in this interconversion. 5. Attempts at partial purification have revealed that the enzyme resembles the pyruvate kinase of skeletal muscle, rather than that of liver, in its solubility in ammonium sulphate and elution from DEAE-cellulose. 6. The relevance of these properties in the regulation of pyruvate kinase activity in vivo in adipose tissue is discussed.
\end{abstract}

In tissues and organisms that are capable of active gluconeogenesis, the stage of $\mathrm{PEP} *$ utilization represents an important metabolic control point. Under conditions of hexose phosphate synthesis, pyruvate and $\mathrm{C}_{4}$ acids give rise to PEP through the reactions catalysed by pyruvate carboxylase (EC 6.4.1.1) and PEP carboxykinase (EC 4.1.1.32). It is clear that, from equilibrium considerations alone, any significant simultaneous PyK activity would serve merely to recycle pyruvate at the expense of ATP (Sols, 1968).

It has been demonstrated that PyK from several gluconeogenic sources is strongly and specifically activated at low concentrations of FDP (Gancedo, Gancedo \& Sols, 1967; Hess, Haeckel \& Brand, 1967; Hunsley \& Suelter, 1967; Tanaka, Sue \& Morimura, 1967b; Taylor \& Bailey, 1967; Susor \& Rutter, 1968); it has been postulated that this activation in vitro may reflect a physiological mechanism whereby PyK activity is increased during glycolysis and markedly inhibited during gluconeogenesis (Gancedo et al. 1967; Hess et al. 1967; Tanaka, Harano, Sue \& Morimura, 1967a).

* Abbreviations: PEP, phosphoenolpyruvate; FDP, fructose 1,6-diphosphate; PyK, pyruvate kinase (ATPpyruvate phosphotransferase, EC 2.7.1.40).
Several observations suggest that PyK activity may also be controlled through changes in the total enzyme concentration. Tanaka, Harano, Morimura \& Mori (1965) and Tanaka et al. (1967a) showed that the concentration of the FDP-sensitive PyK (PyK-L) in rat liver is conditioned by the hormonal and nutritional status of the animal (Tanaka et al. $1965,1967 a$ ). Similar fluctuations in concentration have been observed with the enzyme from rat kidney (Krebs \& Eggleston, 1965). In yeasts, both mechanisms (activation and induction) appear to be present (Hommes, 1966; Gancedo et al. 1967; Fernández, Medrano, Ruiz-Amil \& Losada, 1967), though not necessarily together in any one species (Gancedo et al. 1967).

Earlier reports suggested that adipose tissue contains pyruvate carboxylase activity (Wise \& Ball, 1964; Ballard \& Hanson, 1967), but is deficient in PEP carboxykinase (Young, Shrago \& Lardy, 1964; Weber, Hird, Stamm \& Wagle, 1965). Ballard, Hanson \& Leveille (1967) and Reshef, Niv \& Shapiro $(1967 a, b)$, however, produced evidence that both pyruvate and propionate are effective precursors of L-glycerol 3-phosphate in this tissue, especially when the availability of glucose is limited. For pyruvate incorporation, the 
distribution of ${ }^{14} \mathrm{C}$ in glyceride glycerol was consistent with a pathway involving an active dicarboxylic acid shuttle (Ballard et al. 1967). Further, more sensitive assay methods showed that PEP carboxykinase is present in isolated adipocytes in sufficient concentration to account for the observed rate of conversion of pyruvate into L-glycerol 3-phosphate (Ballard et al. 1967).

Measurements of glycolytic intermediates in epididymal fat pads incubated with glucose and insulin indicated that the PyK reaction in this tissue is far displaced from equilibrium. Under these conditions the concentration of ATP was $10 \mathrm{~mm}$, that of ADP $5 \mathrm{~mm}$, that of PEP $0.03 \mathrm{~mm}$ (minimum value) and that of pyruvate maximally $1.0 \mathrm{~mm}$ (as determined from pyruvate output into the incubation medium) (M. Halperin \& R. M. Denton, personal communication); these data give a maximum steady-state mass-action ratio of 70 , in comparison with the thermodynamic equilibrium constant of 2000-15000 (Williamson, 1965).

In view of the presence of an active dicarboxylic acid shuttle and of the considerations detailed above, it was decided to conduct a detailed investigation of the properties of PyK from adipose tissue. In the present paper, evidence is presented to show that the enzyme exists in two interconvertible forms with distinctive kinetic properties. The effects of the substrates PEP and ADP, the effectors ATP and FDP, and of $\mathrm{K}^{+}$, $\mathrm{NH}_{4}{ }^{+}$and $\mathrm{Mg}^{2+}$, were studied to gain some insight into a possible physiological mechanism of PyK regulation.

A preliminary communication dealing with the interconversion of the two forms, and their electrophoretic and ultracentrifugal properties, has been published (Pogson, 1968).

\section{MATERIALS AND METHODS}

Chemicals and enzymes. ATP (disodium salt), ADP (free acid and trisodium salt), FDP (tetracyclohexylammonium salt), 3-phosphoglycerate (sodium salt) and NADH (sodium salt) were obtained through the Boehringer Corp. (London) Ltd. (London, W.5). Tris (Trizma base) was from the Sigma Chemical Co. (St Louis, Mo., U.S.A.) and bovine serum albumin fraction $\mathrm{V}$ from the Armour Pharmaceutical Co. Ltd. (Eastbourne, Sussex). Imidazole and 2-mercaptoethanol were from Koch-Light Laboratories Ltd. (Colnbrook, Bucks.) and were used without further purification. All inorganic chemicals were of A.R. grade.

PEP (monocyclohexylammonium salt) was a gift from Dr A. J. Kirby. CoA, acetyl-CoA and L-glycerol 3-phosphate were given by $\mathrm{Dr}$ R. M. Denton and palmitoyl-CoA was given by Dr P. B. Garland.

All enzymes were obtained through the Boehringer Corp. (London) Ltd.

Rats. Male albino Wistar rats, fed on a stock laboratory diet (Short \& Parkes, 1949), were used throughout. In two starvation experiments animals weighing $200 \mathrm{~g}$. were used; in all other experiments animals weighing $150-300 \mathrm{~g}$. were used. No differences were noted between experiments in which rats of different age and weight were used.

Buffer solutions. All buffers were adjusted to the appropriate $\mathrm{pH}$ on a glass electrode, either with $\mathrm{N}-\mathrm{NaOH}$ or with $50 \%(\mathrm{v} / \mathrm{v})$ acetic acid.

Dialysis of lactate dehydrogenase (EC 1.1.1.27). In most experiments, lactate dehydrogenase was used untreated. In experiments designed to investigate the effect of univalent cations, however, it was essential to exclude such ions from the basic assay medium.

Dialysis tubing $\left(\frac{8}{32} \mathrm{in}\right.$.) was treated to remove plasticizing substances, as described by Garland, Newsholme \& Randle (1964). A sample (2.0 ml.) of pig heart lactate dehydrogenase (10 mg./ml., 360 units $/ \mathrm{mg}$.) was dialysed against five changes of 11. of $20 \mathrm{~mm}$-imidazole-acetate buffer, $\mathrm{pH} \mathrm{7.4}$, at $4^{\circ}$. The final diffusate gave no precipitate with $\mathrm{BaCl}_{2}-\mathrm{HCl}$. The enzyme was then diluted to $20 \mathrm{ml}$. with $20 \mathrm{~mm}$-imidazole-


$(1 \%) ; 1.0 \mathrm{ml}$. portions were stored at $-15^{\circ}$, and were assayed before use.

Chemical assays. For kinetic experiments, solutions of PEP, ADP, ATP and FDP were standardized by enzymic assay in quintuplicate. All such solutions were stored at $-15^{\circ}$; FDP was kept indefinitely, but PEP, ADP and ATP were discarded after 2 weeks. PEP and ADP were assayed with the system described below for the assay of PyK activity. FDP and ATP were assayed as described by Pogson \& Randle (1966).

Standard solutions of $\mathrm{KCl}, \mathrm{NH}_{4} \mathrm{Cl}$ and $\mathrm{MgCl}_{2}$ were made up by dissolving known weights of solid in glass-distilled water; $\mathrm{NH}_{4} \mathrm{Cl}$ and $\mathrm{MgCl}_{2}$ solutions were brought to $\mathrm{pH} 6 \cdot 8-$ $7 \cdot 0$ with $\mathrm{N} \cdot \mathrm{NaOH}$.

ADP (trisodium salt) was found to contain significant amounts of AMP (5-12\%) and of ATP (approx. 1\%). Its use was therefore restricted to assays for total PyK activity. In kinetic experiments, in which it was preferable to exclude both AMP and (especially) ATP, ADP (free acid) was used. This contained less than $1 \%$ of AMP and was free of ATP. The concentration of $\mathrm{Mg}^{2+}$ in solutions of ADP and ATP was measured as described by Garner (1946). ATP solutions contained $3-5 \%$ of $\mathrm{Mg}^{2+}$ on a molar basis and ADP solutions less than $1 \%$.

Extraction of PyK activity. Two to four rats were killed by a blow on the head followed by decapitation. Epididymal fat pads were removed, washed in ice-cold $0.9 \% \mathrm{NaCl}$, blotted, weighed and dispersed in buffer in a hand-operated Potter-Elvehjem homogenizer. Two buffer systems were used: PyK-A activity (see the Results section) was obtained by extraction in 20 mM-imidazole-acetate-5 mM-EDTA10 mM-2-mercaptoethanol, $\mathrm{pH} \mathrm{6.8,} \mathrm{and} \mathrm{PyK-B} \mathrm{by} \mathrm{extraction}$ in 20 mM-imidazole-acetate, $\mathrm{pH} 6 \cdot 8$. The homogenates were spun at $40000 \mathrm{~g}$ for $30 \mathrm{~min}$. at $2^{\circ}$ in an MSE High Speed 18 centrifuge. After removal of the fat plug, the supernatant was used for all experiments without further purification.

In severalinstances, extraction in imidazole-acetate alone yielded PyK-B containing significant amounts of PyK-A. Since, for kinetic studies, any contribution by PyK-A was undesirable, such extracts were incubated for $20 \mathrm{~min}$. at $34^{\circ}$ with $25 \mu \mathrm{M}$-FDP. Under these conditions all PyK-A was converted into PyK-B (see below). The presence of this low concentration of FDP did not noticeably affect rates in subsequent assays. 
Assay of total PyK activity. PyK activity was assayed at $25^{\circ}$ with a $1 \mathrm{~cm}$. light-path in a Hilger-Gilford recording spectrophotometer. The assay medium contained: $0 \cdot 125 \mathrm{M}$ tris-acetate buffer; $12.5 \mathrm{~mm}-\mathrm{MgCl}_{2} ; 0.15 \mathrm{M}-\mathrm{KCl} ; 2 \mathrm{mM}-\mathrm{ADP}$; 1.5 mм-PEP; $0.125 \mathrm{~mm}-\mathrm{NADH} ; \quad 0.3 \%$ bovine serum albumin; 3.6units of lactate dehydrogenase: the final $\mathrm{pH}$ was 7.4 and the final volume $3.0 \mathrm{ml}$. The reaction was initiated by the addition of enzyme, and the decrease in $E_{340}$ was followed by using a full-chart scale width of $0 \cdot 1 E$ unit. The rates were linear over at least 5-10 min., and were directly proportional to the volume of enzyme added. One unit of PyK catalyses the disappearance of $1 \mu$ mole of PEP $/$ min. at $25^{\circ}$.

Blank determinations without ADP were carried out simultaneously. NADH oxidase activity, including nonspecific phosphatase activity, was between $0.2 \%$ and $0.7 \%$ of the total PyK activity.

Kinetic experiments. The activity was followed in a system similar to that described above, but at $30^{\circ}$ and with various concentrations of ADP, PEP and cations. At sub-optimum concentrations of PEP, rates with PyK-A were linear. With PyK-B under similar conditions, rates fell off markedly after an initial linear phase of approx. 1 min.; experimental rates were measured over the initial period.

In experiments with FDP, the apparent blank NADH oxidase activity was enhanced owing to the presence in the extracts of aldolase (EC 4.1.2.7), triose phosphate isomerase (EC 5.3.1.1) and L-glycerol 3-phosphate dehydrogenase (EC 1.1.1.8). In these instances, the measurements of the rates in both blank and experimental cuvettes were carried out in triplicate. The blank rates, which were highly reproducible, varied between $2 \%$ (at high PEP concentrations) and 30\% (at the lowest PEP concentration) of the corresponding experimental rates.

The extracts were also found to contain significant adenylate kinase (EC 2.7.4.3) activity. Although without effect in the lactate dehydrogenase assay system, this activity precluded measurements of PyK activity through hexokinase (EC 2.7.1.1) and glucose 6-phosphate dehydrogenase (EC 1.1.1.49).

Incubation experiments. PyK-A and PyK-B were readily distinguished by their respective reaction rates at low PEP concentrations (see Fig. 1). Two assays were used. In both the PEP concentration was $0.03 \mathrm{~mm}$; the ADP concentration was either $0.25 \mathrm{~mm}$ or $2.0 \mathrm{mM}$. The latter assay system was devised for use in experiments involving incubation with ATP where blank adenosine triphosphatase activity might substantially increase the concentration of ADP and hence interfere with normal assay conditions.

The percentage of PyK-B in a mixture after incubation was calculated from the experimentally determined rates with $100 \%$ PyK-A and 100\% PyK-B as follows:

$$
\begin{aligned}
& \% \text { of PyK-B } \\
& =\frac{(\text { observed rate })-(\text { rate with PyK-A) }}{(\text { rate with PyK-B) }-(\text { rate with PyK-A) }} \times 100
\end{aligned}
$$

The two extreme activities were produced by preliminary incubation for $20 \mathrm{~min}$. at $34^{\circ}$ of portions of the initial extract with respectively 33.3 mm-EDTA (for $100 \%$ PyK-A) or 0.5 mM-FDP (for $100 \%$ PyK-B).

Experimental incubations were carried out for $10 \mathrm{~min}$. at $34^{\circ}$ with $0.2 \mathrm{ml}$. of extract in a final volume, with additions, of $0.3 \mathrm{ml}$. After incubation, tubes were kept at $0^{\circ}$ and were assayed as soon as possible.

Expression of results. Reaction velocities $(v)$ throughout have been corrected for enzyme activity and are expressed as $\mu \mathrm{moles} / \mathrm{min}$./unit of PyK at $25^{\circ}$. The lines drawn in the Figures represent the best fit by eye.

\section{RESULTS}

Effect of PEP concentration. Preliminary experiments in which the tissue extractions were carried out in buffers with and without 5mm-EDTA indicated that the behaviour of the extracts towards PEP was markedly influenced by EDTA. Fig. 1 shows the relationship between reaction velocity and PEP concentration in the two cases. In the absence of EDTA, 'normal' Michaelis-Menten kinetics were observed, with $K_{m}$ for PEP $67 \mu \mathrm{M}$. This compares with values of $75 \mu \mathrm{M}$ for rabbit muscle PyK (Kerson, Garfinkel \& Mildvan, 1967) and of $67 \mu \mathrm{M}$ for rat heart PyK (C. I. Pogson, unpublished work). When EDTA was present, no significant change in $V_{\max }$. was apparent, but the rate-response curve was markedly sigmoid with an apparent $K_{m}$ for PEP of $0.6 \mathrm{~mm}$. These two forms of the enzyme were preliminarily designated PyK-A (extracted with EDTA, sigmoid response with PEP) and PyK-B (extracted in the absence of EDTA, Michaelis-Menten kinetics with PEP) (Pogson, 1968). The large difference between the rates observed with the two forms at PEP concentrations of $0.1 \mathrm{~mm}$ and below provided a useful assay system whereby the proportions of both forms in a mixture can be satisfactorily determined (see below, and the Materials and Methods section).

Effect of FDP. The effect of FDP on the activity of PyK-A at various PEP concentrations is also shown in Fig. 1. Although at concentrations above $0.25 \mathrm{~mm}$-PEP FDP was without apparent effect, at PEP concentrations in the physiological range

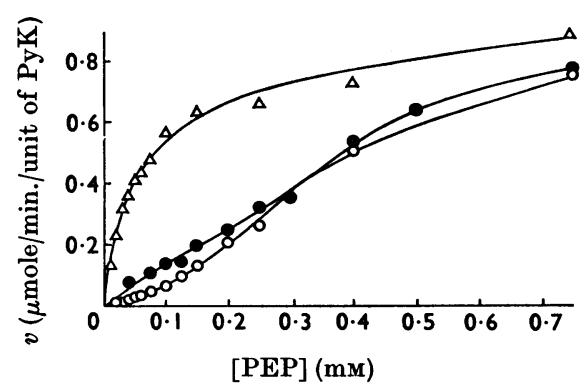

Fig. 1. Activity of the two forms of adipose-tissue PyK with various concentrations of PEP. The assay system was as described in the text, with $0.15 \mathrm{M}-\mathrm{KCl}$ and $0.25 \mathrm{~mm}-\mathrm{ADP}$. O, PyK-A (extracted with EDTA); •, PyK-A plus $3.0 \mathrm{~mm}$ FDP; $\triangle$, PyK-B (extracted in the absence of EDTA). 


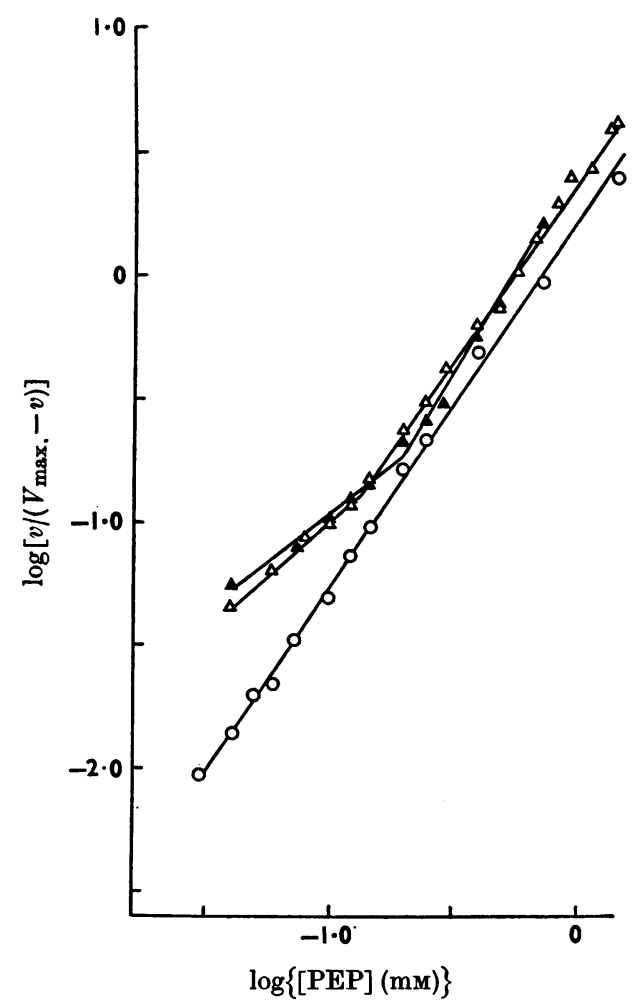

Fig. 2. Hill plots for PyK-A with various concentrations of PEP with and without FDP. The assay system was as described in the text, with $0 \cdot 15 \mathrm{M}-\mathrm{KCl}$ and $0.25 \mathrm{~mm}-\mathrm{ADP}$. FDP concentrations: $O$, none; $\triangle, 0.5 \mathrm{~mm} ; \Delta, 3.0 \mathrm{~mm}$. (approx. $0.05 \mathrm{~mm}$ in adipose-tissue intracellular water; R. M. Denton \& M. Halperin, personal communication) marked activation was observed. The control ratio (activated rate/basal rate) varied between 1 and 10 over the range measured; these values were much lower than those reported for the same enzyme from other sources (Hess et al. 1967; Taylor \& Bailey, 1967). Hill plots \{plots of $\log \left[v /\left(V_{\max }-v\right)\right]$ against $\left.\log s\right\}$ demonstrating this effect of FDP more clearly are shown in Fig. 2. With PEP alone, the slope $n$ varied between 1.4 and 1.8 ; in the presence of FDP $n$ values approximated to unity only at low PEP concentrations. Fig. 3 shows the effect of increasing the concentration of FDP at a possible physiological concentration of PEP $(0.03 \mathrm{~mm})$. The kinetics obeyed the Michaelis-Menten relationship with an $n$ value of unity, in contrast with the effect of FDP on yeast PyK (Hess et al. 1967). A modified LineweaverBurk plot of these data (Fig. 3, inset) gave a $K_{a}$ value for FDP of $29 \mu \mathrm{M}$, somewhat higher than values reported for PyK from other sources (Taylor \& Bailey, 1967; Susor \& Rutter, 1968).

FDP was without similar effect on PyK-B under all the conditions tested.

Effect of ADP concentration. The kinetic behaviour of both PyK-A and PyK-B with respect to ADP and PEP was consistent with random binding of the two substrates, in agreement with the findings of Reynard, Hass, Jacobsen \& Boyer (1961), and in contrast with the findings of Koler et al. (1963) for the anomalous PyK from erythrocytes.

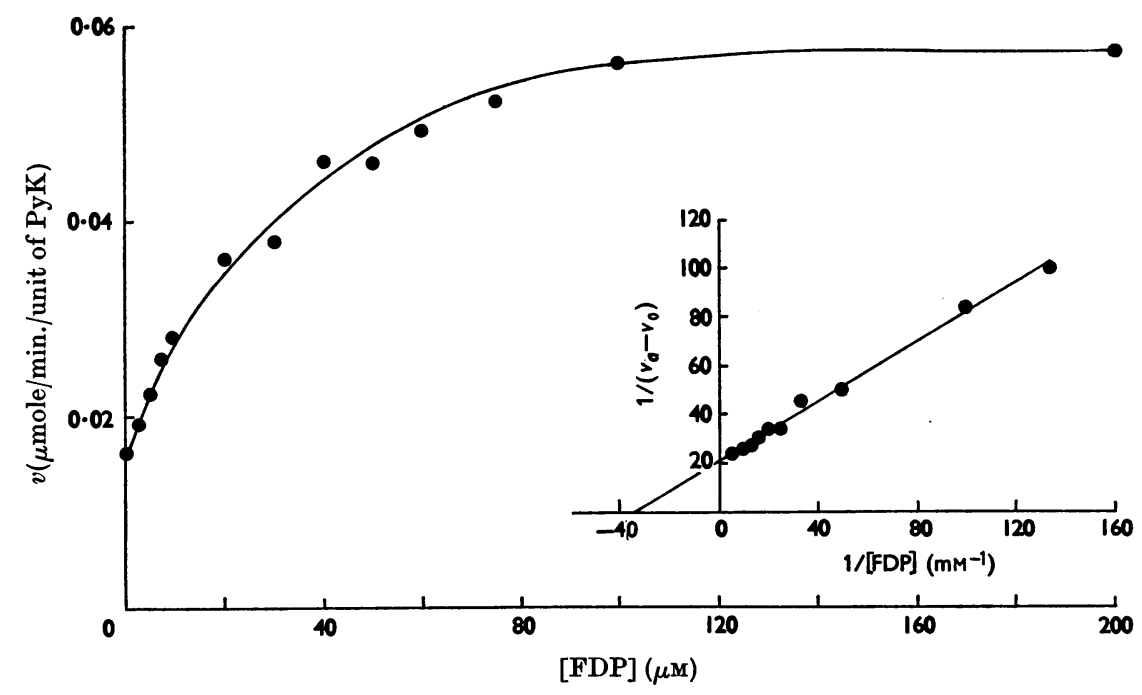

Fig. 3. Effect of FDP on rates of PyK-A at a near-physiological concentration of PEP (0.03 mM). The assay system was as described in the text, with $0.15 \mathrm{M}-\mathrm{KCl}$ and $0.25 \mathrm{~mm}-\mathrm{ADP}$. The inset shows a modified LineweaverBurk plot of the same results; $K_{a}$ for FDP was $29 \mu \mathrm{M}$. $v_{0}$, velocity in the absence of FDP; $v_{a}$, velocity with FDP. 

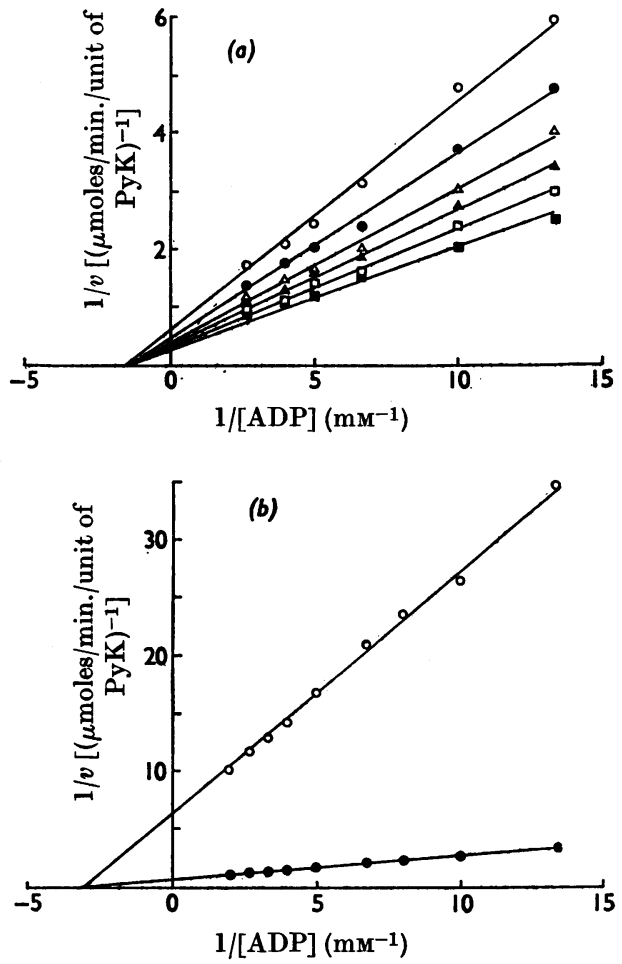

Fig. 4. Double-reciprocal plots for the rates of the two forms of adipose-tissue PyK with various concentrations of ADP and fixed concentrations of PEP. The assay system was as described in the text, with 0.15 M-KCl. (a) PyK-B. The $K_{m}$ for ADP was $0.67 \mathrm{mM}$. PEP concentrations (mM): O $0.03 ; \bullet, 0.04 ; \triangle, 0.05 ; \Delta, 0.06 ; \square, 0.075 ; \square, 0.1$. (b) PyK-A. The $K_{m}$ for ADP was 0.33 mm. PEP concentrations $(\mathrm{mM}): \bigcirc, 0 \cdot 1 ; 0,0 \cdot 5$.

Plots of rate against increasing ADP concentration were of the conventional Michaelis-Menten form for both PyK-A and PyK-B. Fig. 4 shows Lineweaver-Burk plots at different PEP concentrations: the $K_{m}$ values for ADP were $0.33 \mathrm{~mm}$ and 0.67 mM for PyK-A and PyK-B respectively.

Effect of univalent cations. Both forms of adiposetissue PyK exhibit an absolute requirement for a univalent cation. The response to $\mathrm{K}^{+}$and $\mathrm{NH}_{4}{ }^{+}$ was investigated; these ions could not be replaced by $\mathrm{Na}^{+}$, which was totally without effect either as an activator or as an inhibitor of $\mathrm{K}^{+}$binding.

The response of PyK-A and PyK-B towards increasing concentrations of $\mathrm{K}^{+}$and $\mathrm{NH}_{4}{ }^{+}$in the presence of various concentrations of PEP and ADP is shown in Fig. 5. Plots with both forms of the enzyme exhibited pronounced sigmoid characteristics, with Hill-plot $n$ values between 1.9 and 2.0 for PyK-A and between 1.4 and 1.75 for
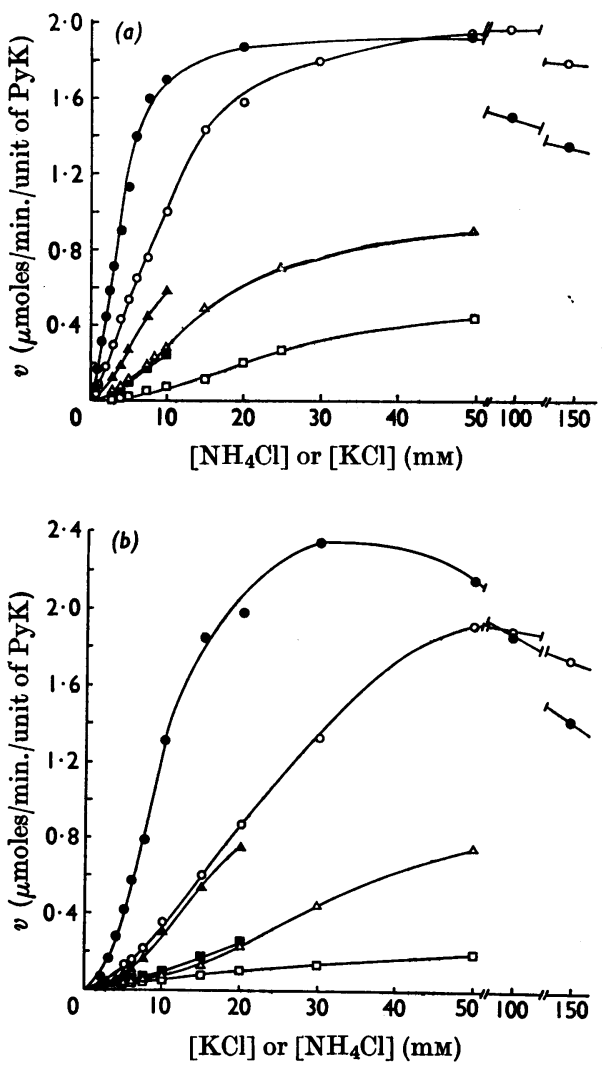

Fig. 5. Effect of univalent cations on the activities of the two forms of adipose-tissue PyK. The assay conditions were as given in the text; $\left(\mathrm{NH}_{4}\right)_{2} \mathrm{SO}_{4}$ was previously removed from the lactate dehydrogenase by dialysis. Open symbols represent rates with $\mathrm{K}^{+}$; filled-in symbols represent those with $\mathrm{NH}_{4}{ }^{+}$. (a) PyK-B. $\mathrm{O}$ and $0,1.0 \mathrm{mM}-\mathrm{PEP}$, 2.0mM-ADP; $\triangle$ and $\Delta, 0.5 \mathrm{~mm}-\mathrm{PEP}, \quad 0.25 \mathrm{~mm}-\mathrm{ADP}$; $\square$ and $\square, 0.05 \mathrm{~mm}-\mathrm{PEP}, 0.25 \mathrm{~mm}-\mathrm{ADP}$. (b) PyK-A. $O$ and - 1.0 mM-PEP, 2.0 mM-ADP; $\triangle$ and $\triangle, 1.0 \mathrm{mM}-\mathrm{PEP}$, 0.25 mM-ADP; $\square$ and $\square, 0.3 \mathrm{~mm}-\mathrm{PEP}, 0.25 \mathrm{~mm}-\mathrm{ADP}$.

PyK-B. $\mathrm{NH}_{4}{ }^{+}$was more effective than $\mathrm{K}^{+}$as an activator of both forms. The apparent $K_{m}$ values for these ions were dependent on the concentrations of substrates, becoming lower with increasing concentrations of PEP and ADP (Table 1).

As noted by Melchior (1965), high concentrations of $\mathrm{K}^{+}$(above $0 \cdot 1 \mathrm{M}$ ) produced some degree of inhibition. A similar effect was also seen with $\mathrm{NH}_{4}{ }^{+}$at a somewhat lower concentration (above $50 \mathrm{~mm}$ ).

With PyK-A alone, and at concentrations of PEP and ADP that fall within the physiological range $(0.06 \mathrm{~mm}$ and $2.0 \mathrm{~mm}$ respectively; Denton, Yorke \& Randle, 1966), $\mathrm{NH}_{4}{ }^{+}$produced an apparent 
fivefold increase in $V_{\max }$ (Fig. 6a); that this may have been due to a reciprocal decrease of the apparent $K_{m}$ for PEP in the presence of $\mathrm{NH}_{4}{ }^{+}$is shown in Fig. 6(b). PyK-B under similar conditions did not show this effect.

The presence of FDP had no effect on the behaviour of either form of the enzyme with univalent cations.

Effect of $\mathrm{Mg}^{2+}$. Fig. 7(a) shows the effect of increasing $\mathrm{Mg}^{2+}$ concentration on the reaction velocity of PyK-A under three different substrate conditions: $n$ values from Hill plots varied between 1.7 and 2.0. The apparent $K_{m}$ for $\mathrm{Mg}^{2+}$ was

Table 1. $K_{m}$ values for $\mathrm{K}^{+}$and $\mathrm{NH}_{4}{ }^{+}$at various concentrations of PEP and ADP

\begin{tabular}{lcccc} 
Enzyme & & & \multicolumn{2}{c}{$K_{m}(\mathrm{mM})$} \\
activity & {$[\mathrm{PEP}](\mathrm{mM})$} & {$[\mathrm{ADP}](\mathrm{mM})$} & $\mathrm{K}^{+}$ & $\mathrm{NH}_{4^{+}}$ \\
PyK-A & 0.06 & 2.0 & 46 & 34 \\
& 0.06 & 3.3 & 36 & \\
& 1.0 & 0.25 & 44 & 18 \\
& 1.0 & 2.0 & 22 & 9 \\
PyK-B & 0.05 & 0.25 & 23 & 10 \\
& 0.5 & 0.25 & 15 & 8 \\
& 1.0 & 2.0 & 10 & 4
\end{tabular}

dependent on PEP concentration alone, decreasing from $4.3 \mathrm{~mm}$ at $0.3 \mathrm{~mm}$-PEP to $2.3 \mathrm{~mm}$ at $1.0 \mathrm{~mm}$ PEP; variations in ADP concentration had no effect.

In contrast, PyK-B exhibited Michaelis-Menten kinetics for rate-response curves with increasing $\mathrm{Mg}^{2+}$ concentration. Fig. $7(b)$ is a Lineweaver-Burk plot showing the effect of PEP concentration on $\mathrm{Mg}^{2+}$ binding. In this instance the $K_{m}$ for $\mathrm{Mg}^{2+}$ increased from $1.4 \mathrm{mM}$ to $2.7 \mathrm{mM}$ as the PEP concentration was raised from $0.05 \mathrm{mM}$ to $1.0 \mathrm{mM}$; ADP was again without effect.

The interaction between $\mathrm{Mg}^{2+}$ and PyK-A remains unaffected by the presence of FDP at saturating concentrations.

Inhibition by $A T P$. In agreement with reports on PyK from other sources (Reynard et al. 1961; Hess et al. 1967; Tanaka et al. 1967a; Susor \& Rutter, 1968) it was found that ATP was a potent inhibitor of both forms of the adipose-tissue enzyme. Fig. 8(a) shows the effect of ATP on PEP rate-response curves for PyK-A. Owing to high blank rates, probably due, at least in part, to adenosine triphosphatase and adenylate kinase activity, quantitative results were not obtained to show ATP competition also with ADP; qualitative experiments showed that such competition is present.

Fig. $8(b)$ is a double-reciprocal plot demonstrating
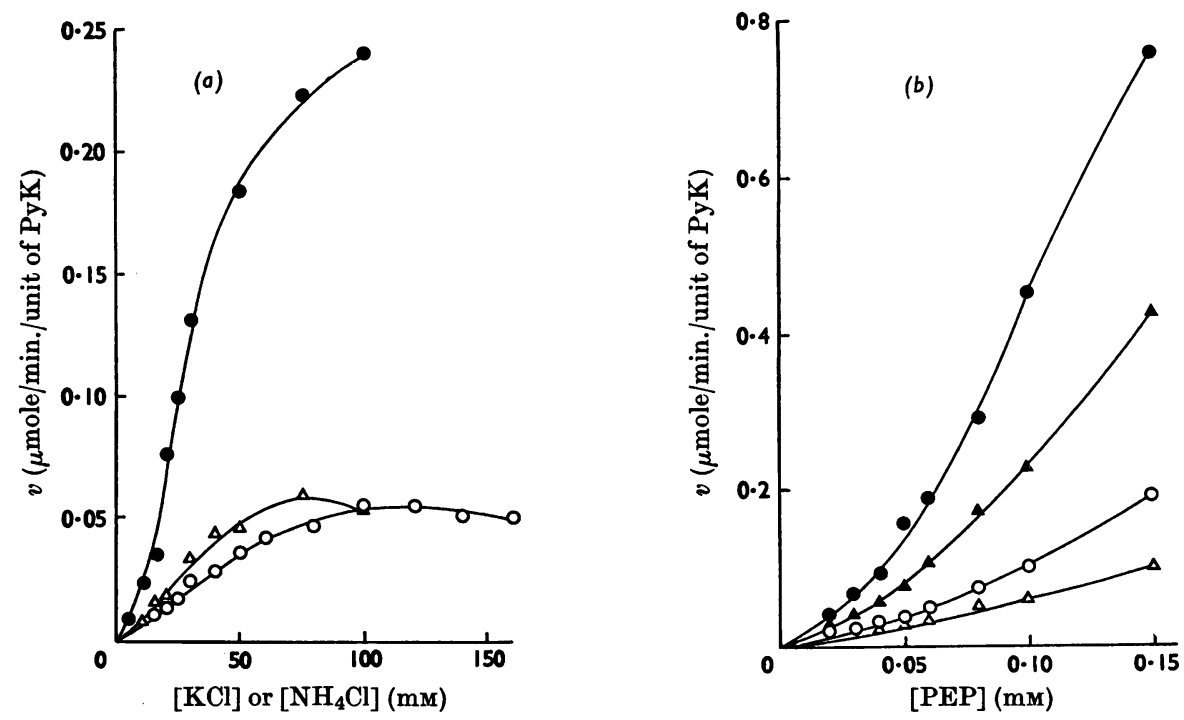

Fig. 6. Effect of univalent cations on the activity of PyK-A at low concentrations of PEP. The assay conditions were as given in the text; $\left(\mathrm{NH}_{4}\right)_{2} \mathrm{SO}_{4}$ was previously removed from the lactate dehydrogenase by dialysis. Open symbols represent rates with $\mathrm{K}^{+}$; filled-in symbols represent those with $\mathrm{NH}_{4}{ }^{+}$. (a) Rates with various concentrations of $\mathrm{K}^{+}$and $\mathrm{NH}_{4}{ }^{+}$at a near-physiological concentration of $\mathrm{PEP}(0.06 \mathrm{~mm})$. $O$ and $\bullet, 2 \cdot 0 \mathrm{mM}-\mathrm{ADP} ; \Delta, 3.3 \mathrm{mM}-$ ADP. (b) Rates at various concentrations of PEP with saturating concentrations of $\mathrm{K}^{+}(0 \cdot 15 \mathrm{M}) \mathrm{or}^{\mathrm{NH}} \mathrm{N}^{+}(0 \cdot 1 \mathrm{M})$. $O$ and $0,2 \cdot 0 \mathrm{~mm}-\mathrm{ADP} ; \triangle$ and $\triangle, 0.25 \mathrm{~mm}-\mathrm{ADP}$. 

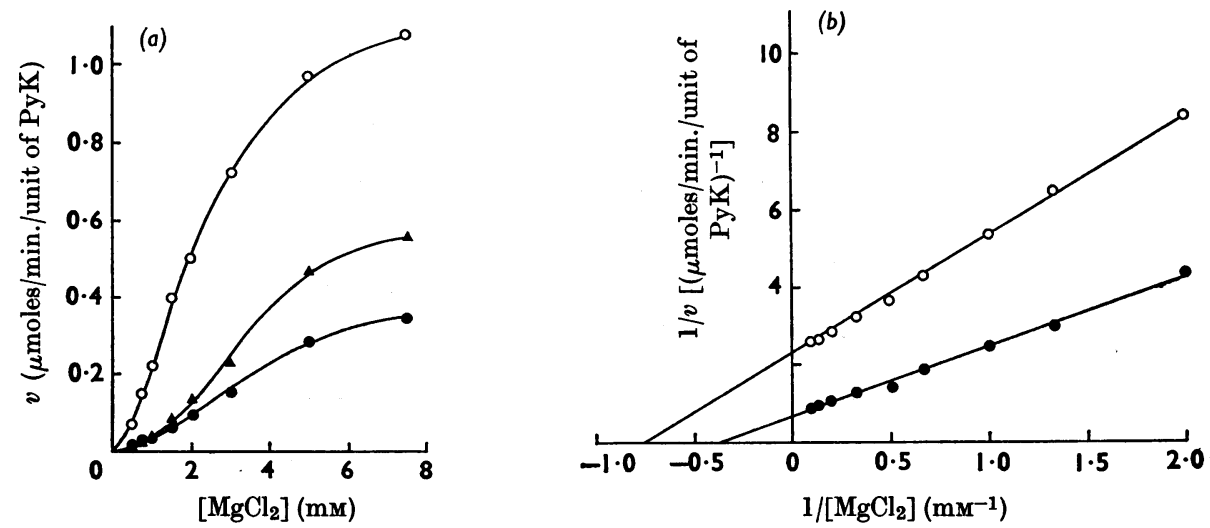

Fig. 7. Effect of $\mathrm{Mg}^{2+}$ concentration on the activity of the two forms of adipose-tissue PyK. The assay conditions were as described in the text, with 0.15 M-KCl. (a) PyK-A. O, 1.0mM-PEP, 0.25 mM-ADP; 0, 0.3 mM-PEP, 0.25 mM-ADP; $\triangle, 0.3 \mathrm{~mm}-\mathrm{PEP}, 2.0 \mathrm{~mm}-\mathrm{ADP}$. (b) PyK-B. Double-reciprocal plot. The concentration of ADP

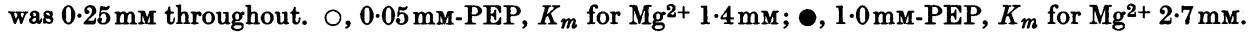


Fig. 8. Inhibitory effect of ATP on the two forms of adipose-tissue PyK. The assay system was as described in the text with $0.15 \mathrm{M}-\mathrm{KCl}$ and $2.0 \mathrm{~mm}$-ADP. ATP concentrations: O, none;, $2.5 \mathrm{mM} ; \Delta, 5.0 \mathrm{~mm} ; \Delta, 7.5 \mathrm{mM}$. (a) PyK-A. The inset is an enlargement to show rates at low concentrations of PEP. (b) PyK-B. Double-reciprocal plot.

$$
v=\frac{V_{\max .}}{1+\frac{K_{\mathrm{PEP}}}{[\mathrm{PEP}]}+\frac{K_{\mathrm{ADP}}}{[\mathrm{ADP}]}+\frac{K_{\mathrm{PEP}} K_{\mathrm{ADP}}}{[\mathrm{PEP}][\mathrm{ADP}]}\left(1+\frac{[\mathrm{ATP}]}{K_{\mathrm{ATP}}}\right)}
$$

similar effects of ATP on PyK-B activity. To determine meaningful $K_{i}$ values for ATP, the results from Fig. $8(b)$ were fitted to several possible rate equations. A good fit was obtained only with a modified form of the equation derived by Reynard et al. (1961) for rabbit muscle PyK (eqn. 1).

In view of the relatively high $K_{m}$ values for $\mathrm{Mg}^{2+}$ in this instance, it was necessary to expand eqn. (1) to include a term for the effect of this ion (see eqn. 2); due allowance was made also for the removal of free $\mathrm{Mg}^{2+}$ by chelation with ATP (Phillips, George \& Rutman, 1966). Since ADP does not affect the kinetics of $\mathbf{M g}^{2+}$ binding (see above), no account was taken of binding to this nucleotide.

By substitution in eqn. (2), where $V_{\max }$. is the velocity obtained by extrapolation to saturating concentrations of PEP, ADP and $\mathrm{Mg}^{2+}$, and [ATP] 


$$
v=\frac{V_{\max }}{\left\{1+\frac{K_{\mathrm{PEP}}}{[\mathrm{PEP}]}+\frac{K_{\mathrm{ADP}}}{[\mathrm{ADP}]}+\frac{K_{\mathrm{PEP}} K_{\mathrm{ADP}}}{[\mathrm{PEP}][\mathrm{ADP}]}\left(1+\frac{[\mathrm{ATP}]}{K_{\mathrm{ATP}}}\right)\right\}\left(1+\frac{K_{\mathrm{Mg}^{2+}}}{\left[\mathrm{Mg}^{2+}\right]}\right)}
$$

$$
v=\frac{V_{\text {max. }}}{\left\{1+\frac{\left(K_{\mathrm{PEP}}\right)^{n_{1}}}{[\mathrm{PEP}]^{n_{1}}}+\frac{K_{\mathrm{ADP}}}{[\mathrm{ADP}]}+\frac{\left(K_{\mathrm{PEP}}\right)^{n_{1}} K_{\mathrm{ADP}}}{[\mathrm{PEP}]^{n_{1}}[\mathrm{ADP}]}\left(1+\frac{[\mathrm{ATP}]^{n_{3}}}{\left(K_{\mathrm{ATP}}\right)^{n_{2}}}\right)\right\}\left(1+\frac{\left(K_{\mathrm{Mg}^{2+}}\right)^{n_{2}}}{\left[\mathrm{Mg}^{2+}\right]^{n_{2}}}\right)}
$$

Table 2. Compounds tested and found inactive with adipose-tissue PyK

$\begin{array}{lc} & \begin{array}{c}\text { Maximum concn. } \\ (\mathrm{mM})\end{array} \\ \text { Glucose 6-phosphate } & 2 \cdot 0 \\ \text { Fructose 6-phosphate } & 1 \cdot 0 \\ \text { 6-Phosphogluconate } & 0 \cdot 5 \\ \text { L-Glycerol 3-phosphate } & 0 \cdot 2 \\ \text { Citrate } & 2 \cdot 0 \\ \text { 2-Oxoglutarate } & 0 \cdot 4 \\ \text { Palmitate } & 0 \cdot 1^{*} \\ 5^{\prime} \text {-AMP } & 2 \cdot 0 \\ 3^{\prime}, 5^{\prime} \text {-(cyclic)-AMP } & 0 \cdot 2 \\ \text { CoA } & 0 \cdot 2 \\ \text { Acetyl-CoA } & 0 \cdot 14 \\ \text { Palmitoyl-CoA } & 0 \cdot 02 \dagger \\ \text { Phosphate } & 3 \cdot 0 \\ \text { NAD } & 0 \cdot 5 \\ \text { NADH } & 0 \cdot 1 \\ \text { NADP+ } & 0 \cdot 5 \\ \text { NADPH } & 0 \cdot 06 \\ \text { Na } \mathrm{SO}_{4} & \mathbf{3} \cdot 0 \\ \text { * In 5\% bovine serum albumin, fraction } \mathrm{V} . \\ \text { † Tested in the absence of bovine serum albumin. }\end{array}$

represents the total ATP concentration, almost completely present in the form ATPMg ${ }^{2-}$ (Phillips et al. 1966), $K_{i}$ values for ATP binding to PyK-B of $1 \cdot 2-1 \cdot 8 \mathrm{~mm}$ were obtained.

To determine $K_{i}$ for ATP in the PyK-A system, eqn. (2) was further modified to include terms defining the sigmoidicity of the rate curves with PEP, $\mathrm{Mg}^{2+}$ and ATP (not shown). In eqn. (3), $K$ values are the apparent $K_{m}$ values obtained from Hill plots, and $n_{1}, n_{2}$ and $n_{3}$ represent the slopes of Hill plots for PEP, $\mathrm{Mg}^{2+}$ and ATP (1.75, 1.7 and 1.8 respectively). Substitution of the data of Fig. $8(a)$ into this equation yields $K_{i}$ values for ATP of 1.37-1.75 mM.

Effect of other metabolites. A number of other metabolites were tested for activity against both PyK-A and PyK-B. Passeron, Jiménez de Asua \& Carminatti (1967) reported that $\mathrm{Cu}^{2+}$ was a potent inhibitor of rat liver PyK. $\mathrm{Cu}^{2+}$ at $0.02 \mathrm{~mm}$ produced strong inhibition of PyK-A activity, and it was slightly inhibitory even at $2 \mu \mathrm{M}$ in the absence of 2-mercaptoethanol. PyK-B did not show similar sensitivity to $\mathrm{Cu}^{2+}$. 2-Mercaptoethanol protected PyK-A, which may possess a more labile thiol group than does PyK-B (cf. Tanaka et al. 1967a). Weber, Lea \& Stamm (1967) found that acetyl-CoA was inhibitory towards liver PyK; at concentrations up to $0.14 \mathrm{~mm}$ this metabolite was without effect on both the adipose-tissue PyK activities.

None of the other metabolites or ions tested had any action on reaction rates at low PEP concentrations or on the FDP activation of PyK-A. A list of those tested is given in Table 2.

Attempts at further purification. Several attempts were made to separate PyK from contaminating blank activities. In contrast with the behaviour of FDP-sensitive liver PyK (Tanaka et al. 1967a; C. I. Pogson, unpublished work), activity was not retained on DEAE-cellulose equilibrated in $20 \mathrm{~mm}$ tris - acetate - $5 \mathrm{mM}$ - magnesium chloride- $15 \mathrm{~mm}$ potassium chloride buffer, pH 7.4. Further concentration by precipitation with neutral ammonium sulphate led to a remarkable loss of activity; in various experiments, recoveries were as low as $10 \%$. This residual activity was precipitated at $50-60 \%$ saturation; no other fraction contained significant PyK activity.

In view of the possibility of denaturation, interconversion of the two forms, and of the continuing presence of blank activities, it was most satisfactory to carry out the kinetic and other studies with the high-speed supernatant from the adipose-tissue homogenates.

Interconversion of $P y K-A$ and $P y K-B$. PyK-A was converted into PyK-B on a short incubation with FDP at $34^{\circ}$; the reverse process took place in the presence of EDTA (Pogson, 1968). Table 3 shows the effect of certain metabolites and ions on the interconversion of the two forms. In Expt. 1, the conversion of PyK-A into PyK-B, mediated by $0.05 \mathrm{~mm}$-FDP, was unaffected by both substrates, PEP and ADP. ATP at a physiological concentration (Denton et al. 1966) inhibited PyK-B formation. In Expt. 2, both potassium chloride and magnesium 
Table 3. Effect of metabolites and ions on the interconversion of PyK activities

The enzyme was prepared by the extraction of adipose tissue with 20 mM-imidazole-acetate-5 mM-EDTA$10 \mathrm{~mm}$-2-mercaptoethanol buffer, $\mathrm{pH} 6.8$, followed by centrifugation. Incubations were for $10 \mathrm{~min}$. at $34^{\circ} ;$ the incubation mixture consisted of $0.2 \mathrm{ml}$. of enzyme plus additions in a total volume of $0.3 \mathrm{ml}$. After incubation, PyK activity was assayed at $30^{\circ}$ with $0.03 \mathrm{~mm}-\mathrm{PEP}$ and either $0.25 \mathrm{~mm}-\mathrm{ADP}$ (Expt. 2) or 2.0 mM-ADP (Expts. 1 and 3 ). The percentage of PyK-B was calculated as described in the text.

\begin{tabular}{|c|c|c|c|}
\hline Expt. no. & Additions & Time (min.) & $\begin{array}{c}\text { Percentage of } \\
\text { PyK-B }\end{array}$ \\
\hline \multirow[t]{8}{*}{1} & Water & 0 & $\mathbf{0}$ \\
\hline & $\operatorname{ATP}(3.7 \mathrm{~mm})$ & $\begin{array}{l}10 \\
10\end{array}$ & $\begin{array}{l}2 \\
0\end{array}$ \\
\hline & ATP (3.7 mM), FDP (0.05 mM) & 10 & 15 \\
\hline & $\operatorname{ADP}(2 \cdot 0 \mathrm{~mm})$ & 10 & 0 \\
\hline & $\operatorname{ADP}(2.0 \mathrm{~mm}), \mathrm{FDP}(0.05 \mathrm{~mm})$ & 10 & 55 \\
\hline & $\operatorname{PEP}(0.05 \mathrm{~mm})$ & 10 & $\mathbf{0}$ \\
\hline & PEP (0.05 mM), FDP (0.05 mM) & 10 & 45 \\
\hline & FDP $(0.05 \mathrm{~mm})$ & 10 & 41 \\
\hline \multirow[t]{9}{*}{2} & Water & $\{0$ & $\mathbf{0}$ \\
\hline & & 10 & 0 \\
\hline & $\mathrm{KCl}(0 \cdot 1 \mathrm{M})$ & 10 & $\mathbf{0}$ \\
\hline & $\mathrm{KCl}(0.1 \mathrm{M}), \mathrm{FDP}(0.05 \mathrm{mM})$ & 10 & 43 \\
\hline & $\mathrm{MgCl}_{2}(10 \mathrm{mM})$ & 10 & 0 \\
\hline & $\mathrm{MgCl}_{2}(10 \mathrm{mM}), \mathrm{FDP}(0.05 \mathrm{~mm})$ & 10 & 72 \\
\hline & $\mathrm{MgCl}_{2}(0 \cdot 1 \mathrm{M})$ & 10 & 0 \\
\hline & $\mathrm{MgCl}_{2}(0.1 \mathrm{M}), \mathrm{FDP}(0.05 \mathrm{~mm})$ & 10 & 84 \\
\hline & FDP $(0.05 \mathrm{mM})$ & 10 & 53 \\
\hline \multirow[t]{5}{*}{3} & Water & $\begin{aligned} 0 \\
10\end{aligned}$ & 0 \\
\hline & $\mathrm{KCl}(0.1 \mathrm{M}), \operatorname{ATP}(3.7 \mathrm{mM}), \operatorname{ADP}(2.0 \mathrm{mM})$ & 10 & 0 \\
\hline & $\mathrm{KCl}(0.1 \mathrm{M}), \operatorname{ATP}(3.7 \mathrm{mM}), \operatorname{ADP}(2.0 \mathrm{mM}), \operatorname{FDP}(0.05 \mathrm{mM}$ & 10 & 24 \\
\hline & $\mathrm{KCl}(0.1 \mathrm{M}), \operatorname{ATP}(3.7 \mathrm{mM}), \operatorname{ADP}(2.0 \mathrm{mM}), \operatorname{FDP}(0.08 \mathrm{mM}$ & 10 & 52 \\
\hline & FDP $(0.05 \mathrm{~mm})$ & 10 & 51 \\
\hline
\end{tabular}

chloride alone were ineffective; in the presence of FDP, $\mathrm{Mg}^{2+}$ potentiated the action of the effector. Expt. 3 demonstrated that FDP retained its capacity as a sensitive regulator of the interconversion in the presence of physiological concentrations of potassium chloride, ATP and ADP.

The inhibitory action of ATP was also shown by citrate at slightly higher non-physiological concentrations (5 mM). The effect of FDP was specific; no other sugar phosphates were active. $\mathrm{Mg}^{2+}$ could be replaced as a potentiator of FDP action by $\mathrm{Mn}^{2+}, \mathrm{Zn}^{2+}$ or $\mathrm{Co}^{2+} ; \mathrm{Cd}^{2+}$ strongly inhibited the interconversion. Neither $\mathrm{pH}$ changes within the range 6.8-8.0 nor the presence or absence of thiols affected the proportion of the two forms.

When preliminary extraction of the tissue was made into a buffer containing imidazole alone, the proportion of PyK-B was found to be somewhat variable. In two experiments the effects of starvation for 3 days were investigated. Homogenates in 20 mM-imidazole-acetate buffer, $\mathrm{pH} 6 \cdot 8$, from control rats on their normal diet showed $20-70 \%$ of PyK-B; those from 3-day-starved animals contained less than $10 \%$ of PyK-B.

\section{DISCUSSION}

The steady-state kinetics of PyK from adipose tissue indicate that this enzyme is distinct from both types of mammalian PyK described hitherto, though certain properties characteristic of both muscle PyK and liver PyK are present. The three types of mammalian PyK activity are compared in Table 4. The kinetic properties of PyK-A more closely resemble those of liver PyK-L in sensitivity towards FDP and in co-operativity with PEP, $\mathrm{K}^{+}$ and $\mathrm{NH}_{4}{ }^{+}$; the properties of PyK-B resemble those of the muscle enzyme. Similar relationships are apparent in the relative electrophoretic mobilities of the two forms (Pogson, 1968).

The $K_{a}$ for FDP of adipose-tissue PyK is high in comparison with that reported for liver PyK-L (Taylor \& Bailey, 1967; Susor \& Rutter, 1968). This difference may be related to the relative steadystate concentrations of FDP in the two tissues in vivo. Burch (1965) reported that, in liver, FDP concentrations may be as low as $0.02 \mu \mathrm{M}$, which may be a reflection of the activity of the fructose 1,6-diphosphatase (EC 3.1.3.11) of low $K_{m}$ in this 
Table 4. Summary of the properties of types of PyK from three mammalian sources

References: (1) Tanaka et al. (1967a); (2) Kachmar \& Boyer (1953); (3) Mildvan \& Cohn (1965); (4) Reynard et al. (1961); (5) Susor \& Rutter (1968); (6) Pogson \& Denton (1967). The other results are from the present work.

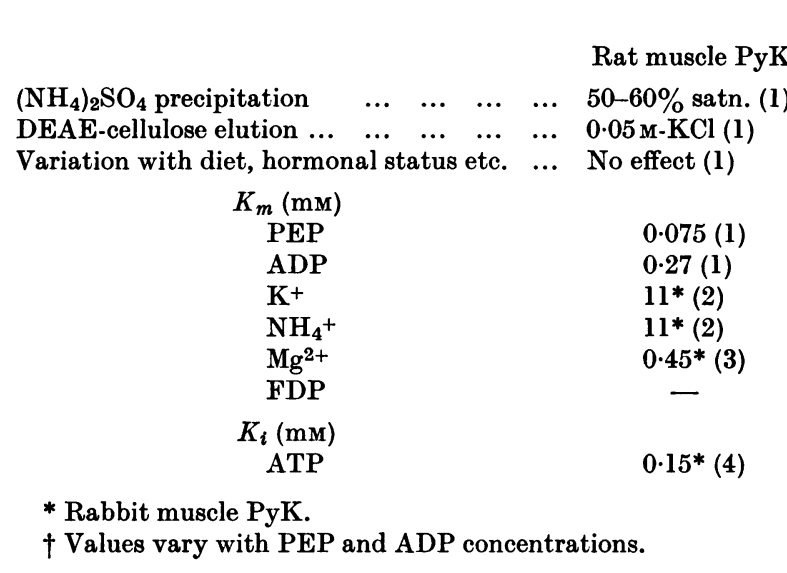

\section{Rat liver PyK (type L)}

$25-45 \%$ satn. (1) $0 \cdot 13 \mathrm{M}-\mathrm{KCl}$ (1)

Effect present (1)

$0 \cdot 84(1)$
$0 \cdot 1(1)$
$0 \cdot 001(5)$

Rat adipose-tissue PyK

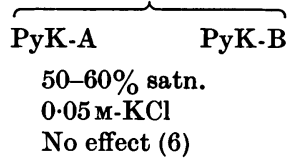

$0 \cdot 6$
$0 \cdot 33$
$20-50 \dagger$
$9-34 \dagger$
$2 \cdot 3-4 \cdot 3 \dagger$
$0 \cdot 03$

$1 \cdot 5$
$0 \cdot 067$

$0 \cdot 67$

10-25†

4-10†

1.4-2.7†

$1 \cdot 5$ tissue. In adipose tissue, in which the gluconeogenic sequence is incomplete owing to the absence of fructose 1,6-diphosphatase (Weber et al. 1965), the concentration of FDP might be expected to be significantly higher. In fat pads incubated with glucose and insulin, FDP concentrations of approx. $0.03 \mathrm{~mm}$ in the intracellular water have been found (M. Halperin \& R. M. Denton, personal communication). These values are consistent with the postulate that FDP is of significance as a controller of PyK activity in adipose tissue in vivo.

The role of inorganic cations in the regulation of metabolic processes remains obscure. In addition to PyK, it is noteworthy that both glucokinase (EC 2.7.1.2) (Parry \& Walker, 1966) and phosphofructokinase (EC 2.7.1.11) (Muntz, 1953; Uyeda \& Racker, 1965) are known to be responsive to change in ionic environment, and in particular to changes in $\mathrm{K}^{+}$concentration. The $K_{m}$ values for $\mathbf{K}^{+}$ binding to PyK, however, are low compared with normal concentrations of intracellular $\mathbf{K}^{+}$(Kernan, 1965); small variations in the concentration of this ion are unlikely to be of any direct regulatory significance in vivo.

The results of Fig. 6 indicate that $\mathrm{NH}_{4}+$ may influence the rates of flow through PyK-A, despite a prevailing high concentration of $\mathrm{K}^{+}$. $\mathrm{NH}_{4}{ }^{+}$ concentrations in rat heart approach $20 \mathrm{~mm}$ (Scharff \& Wool, 1965); similar concentrations in adipose tissue would be consistent with a considerable physiological effect of this ion on the rate of reaction with PyK-A, though not on that with PyK-B.

The action of ATP on adipose-tissue PyK is threefold. First, ATP inhibits competitively with both PEP and ADP at physiological concentrations; secondly, conversion of PyK-B into $\mathrm{PyK}-\mathrm{A}$ is stimulated by increasing ATP concentrations; thirdly, as suggested by England, Denton \& Randle (1967), alterations in ATP concentration may also be reflected in changes in the concentration of 'available' $\mathrm{Mg}^{2+}$, the possible importance of which may be implicit in the co-operative nature of the $\mathrm{Mg}^{2+}-\mathrm{PyK}-\mathrm{A}$ interaction.

The interconversion of PyK-A and PyK-B may theoretically involve an association-dissociation reaction, an intramolecular conformational change, or the gain or loss of a small peptide or other fragment. Ultracentrifugal studies (Pogson, 1968) showed that the change of PyK-A into PyK-B is accompanied by an increase in sedimentation coefficient from $5 \cdot 5 \mathrm{~s}$ to $7 \cdot 2 \mathrm{~s}$. The latter value is similar to those reported for erythrocyte PyK (Koler et al. 1963) and for the active dimer of rabbit muscle PyK (Steinmetz \& Deal, 1966). The change in sedimentation behaviour is insufficient to suggest a true dissociation, and may rather reflect a large conformational change. This would be in keeping with the similar $n$ values for $\mathrm{K}^{+}$and $\mathrm{NH}_{4}+$ binding to the two forms.

The change of PyK-B into PyK-A is occasioned by incubation with EDTA and, possibly more physiologically, ATP or citrate. These compounds resemble each other primarily in being chelators of bivalent cations. Since $\mathbf{M g}^{2+}$ potentiates the effect of FDP in mediating the reverse reaction, it may be inferred that $\mathrm{Mg}^{2+}$ or a similar cation is of direct significance in the interconversion reaction, possibly through binding to both enzyme and FDP.

Tanaka and his co-workers have shown that, in 
liver, PyK-L may be regulated both through alterations of FDP and ATP concentrations and through hormonally induced changes in total enzyme activity (Tanaka et al. 1967a,b). In adipose tissue, under similar conditions, total PyK activity does not alter (Pogson \& Denton, 1967). However, on starvation, the proportion of PyK-B is decreased and that of PyK-A is increased. Such an interconversion would result in a marked diminution in the flow through PyK in vivo. This would be in accord with an increased activity of the 'gluconeogenic' pathway from oxaloacetate to L-glycerol 3-phosphate (Ballard et al. 1967; Reshef et al. $1967 a, b)$. Where the availability of glucose is not limiting, glycolysis provides both L-glycerol 3phosphate and acetyl-CoA for triglyceride synthesis. Under these conditions, the production of FDP through phosphofructokinase may favour PyK-B formation; the properties of this form of the enzyme are further consistent with its role in active glycolysis.

Srere (1967) pointed out the potential hazards of extrapolation from observations in vitro on very dilute enzyme systems to conditions in vivo. This caveat is particularly apposite for adipose-tissue enzymes, which must, of necessity, be subjected to dilutions of at least 100-fold during homogenization. It nevertheless seems reasonable to conclude that the observed properties of fat-pad PyK in vitro are indicative of related functions in vivo; however, certain parameters, such as the rate of interconversion of PyK-A and PyK-B, may be markedly different under the two sets of conditions.

The author is indebted to Dr R. M. Denton, Dr P. B. Garland and Dr A. J. Kirby for their kind gifts of chemicals. Thanks are also due to various members of this Department for helpful discussions, and, in particular, to Professor P. J. Randle for his continued interest and encouragement.

\section{REFERENCES}

Ballard, F. J. \& Hanson, R. W. (1967). J. Lipid Res. 8, 73. Ballard, F. J., Hanson, R. W. \& Leveille, G. A. (1967). J. biol. Chem. 242, 2746.

Burch, H. B. (1965). In Advances in Enzyme Regulation, vol. 3, p. 185. Ed. by Weber, G. Oxford: Pergamon Press Ltd.

Denton, R. M., Yorke, R. E. \& Randle, P. J. (1966). Biochem. J. 100, 407.

England, P. J., Denton, R. M. \& Randle, P. J. (1967). Biochem.J. 105, $32 \mathrm{c}$.

Fernández, M. J., Medrano, L., Ruiz-Amil, M. \& Losada, M. (1967). Europ. J. Biochem. 3, 11.

Gancedo, J. M., Gancedo, C. \& Sols, A. (1967). Biochem. J. $102,23 \mathrm{c}$.

Garland, P. B., Newsholme, E. A. \& Randle, P. J. (1964). Biochem. J. 93, 665.

Garner, R. J. (1946). Biochem. J. 40, 828.
Hess, B., Haeckel, R. \& Brand, K. (1967). Biochem. biophys. Res. Commun. 24, 624.

Hommes, F. A. (1966). Arch. Biochem. Biophys. 114, 231. Hunsley, J. R. \& Suelter, C. H. (1967). Fed. Proc. 26, 559. Kachmar, J. F. \& Boyer, P. D. (1953). J. biol. Chem. 200, 669.

Kernan, R. P. (1965). Cell K, p. 23. London: Butterworths Scientific Publications.

Kerson, L. A., Garfinkel, D. \& Mildvan, A. S. (1967). J. biol. Chem. 242, 2124.

Koler, R. D., Bigley, R. H., Jones, R. T., Rigas, D. A., van Bellinghen, P. \& Thompson, P. (1963). Cold. Spr. Harb. Symp. quant. Biol. 29, 213.

Krebs, H. A. \& Eggleston, L. V. (1965). Biochem. J. 94, 3 c.

Melchior, J. B. (1965). Biochemistry, 4, 1518.

Mildvan, A. S. \& Cohn, M. (1965). J. biol. Chem. 240, 238.

Muntz, J. A. (1953). Arch. Biochem. Biophys. 42, 435.

Parry, M. J. \& Walker, D. G. (1966). Biochem. J. 99, 266.

Passeron, S., Jiménez de Asua, L. \& Carminatti, H. (1967). Biochem. biophys. Res. Commun. 27, 33.

Phillips, R. C., George, P. \& Rutman, R. J. (1966). J. Amer. chem. Soc. 88, 2631.

Pogson, C. I. (1968). Biochem. biophys. Res. Commun. 30, 297.

Pogson, C. I. \& Denton, R. M. (1967). Nature, Lond., 216, 156.

Pogson, C. I. \& Randle, P. J. (1966). Biochem. J. 100, 683.

Reshef, L., Niv, J. \& Shapiro, B. (1967a). J. Lipid Res. 8, 682.

Reshef, L., Niv, J. \& Shapiro, B. (1967b). J. Lipid Res. 8, 688.

Reynard, A. M., Hass, L. F., Jacobsen, D. D. \& Boyer, P. D. (1961). J. biol. Chem. 236, 2277.

Scharff, R. \& Wool, I. G. (1965). Biochem. J. 97, 272.

Short, D. J. \& Parkes, A. S. (1949). J. Hyg., Camb., 47, 209.

Sols, A. (1968). In Carbohydrate Metabolism and its Disorders, vol. 1, p. 79. Ed. by Dickens, F., Randle, P. J. \& Whelan, W. J. London: Academic Press (Inc.) Ltd.

Srere, P. A. (1967). Science, 158, 936.

Steinmetz, M. A. \& Deal, W. C., jun. (1966). Biochemistry, $5,1399$.

Susor, W. A. \& Rutter, W. J. (1968). Biochem. biophys. Res. Commun. 30, 14.

Tanaka, T., Harano, Y., Morimura, H. \& Mori, R. (1965). Biochem. biophys. Res. Commun. 21, 55.

Tanaka, T., Harano, Y., Sue, F. \& Morimura, H. (1967a). J. Biochem., Tokyo, 62, 71.

Tanaka, T., Sue, F. \& Morimura, H. (1967b). Biochem. biophys. Res. Commun. 29, 444.

Taylor, C. B. \& Bailey, E. (1967). Biochem. J. 102, 32 c.

Uyeda, K. \& Racker, E. (1965). J. biol. Chem. 240, 4682.

Weber, G., Hird, H. J., Stamm, N. B. \& Wagle, D. S. (1965). In Handbook of Physiology, Section 5, p. 225. Ed. by Renold, A. E. \& Cahill, G. F., jun. Washington: American Physiological Society.

Weber, G., Lea, M. A. \& Stamm, N. B. (1967). Life Sci. 6, 2441.

Williamson, J. R. (1965). J. biol. Chem. 240, 2308.

Wise, E. M. \& Ball, E. G. (1964). Proc. nat. Acad. Sci., Wash., 52, 1255.

Young, J. W., Shrago, E. \& Lardy, H. A. (1964). Biochemistry, 3, 1687. 